\title{
Nonlinear force-free magnetic field extrapolation in AR 11158 by GPU-DBIE
}

\author{
Rui Wang ${ }^{1}$ and Yihua Yan ${ }^{1}$
}

${ }^{1}$ Key Laboratory of Solar Activity, National Astronomical Observatories, Chinese Academy of Sciences, Beijing 100012, China. email: Ray@nao.cas.cn, yyh@nao.cas.cn

\begin{abstract}
A GPU-based acceleration for the direct boundary integral equation method (GPUDBIE) to extrapolate solar coronal magnetic fields is developed, which is about 1000 times faster than the original DBIE. The 3-d coronal magnetic field is reconstructed for NOAA 11158 on 14-Feb-2011 with the SDO/HMI vector magnetogram as bottom boundary condition. The extrapolated results agree well with the projected SDO/AIA, EUV loops and the STEREO EUV sideviews, which verifies the correctness of our GPU-DBIE method. It is also found that the group of bright EUV loops along magnetic neutral lines agree well with current lines, which may have played an important role in the flare process of the active region.
\end{abstract}

Keywords. active region, corona, GPU, magnetic fields extrapolation

The BIE method (Yan \& Sakurai 2000) or the DBIE method (Yan \& Li 2006) for nonlinear force-free field (NLFFF) extrapolations does not need the arbitrarily-prescribed lateral or side boundary conditions and should be suitable for high-performance parallel computing. However, in the previous work, the implementation of DBIE with parallel computing on high performance computers has not been carried out. Therefore it would
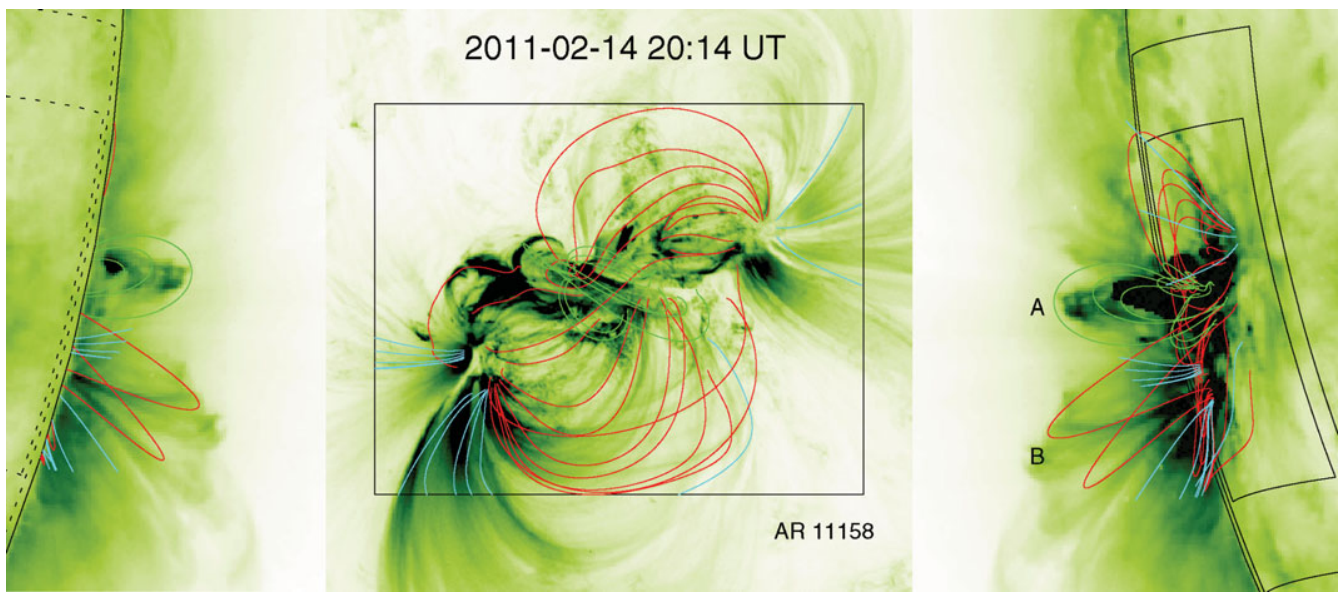

Figure 1. The comparisons of extrapolated results and observed EUV loops. The middle panel is the image from AIA $171 \AA$ band, and the right and left ones from STEREO A and B spacecraft, respectively. The red lines show the closed extrapolated field lines, the light blue lines are the open fields to the computing box and the green lines present the electric current lines. "A" in the right panel indicates a group of EUV bright loops along neutral lines, and "B" another group of high-lying loops. The black square in the middle image presents the $250 \times 200 \times 160$ computational grid domain of DBIE. The outer square on STEREO A image presents the AIA image area and inner one is the same as the square on the AIA image. The squares on the STEREO B image are presented by dotted lines as they are located at the backside of the Sun. 
be slow when extrapolating NLFFF from boundary data with computing grids compatible with current observations (Wiegelmann 2008).

Recently, with the development of Graphics Processing Units (GPUs), it is possible to implement GPU-based parallel computations for the DBIE program. A GPU is composed of high-performance many-core processors capable of very high computation and data throughput (Zhang et al. 2009). The GPU's powerful parallel computing ability to process the integration operation is applied in the DBIE method. The calculation is operated through Intel CPU @ 3.40 GHZ and NVIDIA Geforce GTX 480 graphics device with NVIDIA CUDA 4.2 on a computer. Numerical tests indicate that the GPU-based DBIE program is accelerated almost 1000 times faster as compared with original DBIE. Further acceleration could be achieved through PC-clusters.

In the DBIE method for any point $\mathbf{r}_{i}$, it needs to iterate pseudo force free factor $\lambda_{i}$, which is dependent on $\mathbf{r}_{i}$ only locally, from a compound function (e.g., Eqs.(20) and (22) in Yan \& Li 2006) with the form like $F\left(\lambda_{i}\right)=F\left(\mathbf{B}\left(\lambda\left(\mathbf{r}_{i}\right), \mathbf{r}_{i}\right), \partial \mathbf{B}\left(\lambda\left(\mathbf{r}_{i}\right), \mathbf{r}_{i}\right), \lambda\left(\mathbf{r}_{i}\right), \mathbf{r}_{i}\right)$. In the numerical iteration procedure, as the function $F$ is evaluated at the infinitesimal neighborhood $\pm \delta$ of the point $\mathbf{r}_{i}=\left(x_{i}, y_{i}, z_{i}\right)$, with $\delta$ a very small positive fraction, the zeroth-order approximation has been adopted. So $\lambda\left(\mathbf{r}^{(n)}\right) \approx \lambda_{i}^{(n)}$ over the infinitesimal neighborhood of $\mathbf{r}_{i}$ and the next iteration is obtained from $F\left(\lambda_{i}^{n+1}\right)=$ $F\left(\mathbf{B}\left(\lambda_{i}^{(n)}, \mathbf{r}_{i}\right), \partial \mathbf{B}\left(\lambda_{i}^{(n)}, \mathbf{r}_{i}\right), \lambda_{i}^{(n)}, \mathbf{r}_{i}\right)$. This is a practical and rigorous numerical procedure as opposite to the comments in Rudenko \& Myshyakov (2009).

There have been several studies on the coronal magnetic field reconstruction of the 14-Feb-2011 event in NOAA 11158 (Sun et al. 2012, Wiegelmann et al. 2012). Here the proposed GPU-based DBIE is applied to reconstruct the coronal magnetic field with the vector magnetogram taken at 20:14 UT from the SDO/HMI, as the boundary data pre-processed by the method in Wang et al. (2001). Fig. 1 shows the extrapolated field lines super-imposed on the EUV images by SDO/AIA, and the twin STEREO spacecraft for top- and side-views. The electric current lines are also plotted and they have a good agreement with the central bright EUV loops along neutral lines (A), which may have played an important role in the flare-CME process of the event. From the comparison with extrapolation lines, the highest part of EUV loops (B) is about $86 \mathrm{Mm}$, or $\sim 40 \%$ of the length of the computational domain, in which region the DBIE can achieve very high numerical accuracy.

\section{Acknowledgements}

This work is supported by NSFC Grants No. 10921303, 11273030, MOST Grant No. 2011CB811401, and the National Major Scientific Equipment R\&D Project ZDYZ2009-3. Part of experiments were implemented on the ScGrid and GPU cluser of Supercomputing Center, Computer Network Information Center of Chinese Academy of Sciences.

\section{References}

Rudenko, G. V. \& Myshyakov, I. I. 2009, Solar Phys., 257, 287

Sun, X., Hoeksema, J. T., Liu, Y., Wiegelmann, T., Hayashi, K., Chen, Q., \& Thalmann, J. 2012, ApJ, 748, 77

Wang, H., Yan, Y., \& Sakurai, T. 2001, Solar Phys., 201, 323

Wiegelmann, T. 2008, J. of Geophys. Res. (Space Physics), 113, $03 \mathrm{~s} 02$

Wiegelmann, T., Thalmann, J. K., Inhester, B., Tadesse, T., Sun, X., \& Hoeksema, J. T. 2012, Solar Phys., tmp, 67

Yan, Y. \& Li, Z. 2006, ApJ, 638, 1162

Yan, Y. \& Sakurai, T. 2000, Solar Phys., 195, 89

Zhang, S., Zhu, Y., Zhao, K., \& Zhang, Y. 2009, The High Performance Computing of GPU: CUDA, China Water \& Power Press 